\title{
Orai1 and Stim1 Mediate the Majority of Store-Operated Calcium Entry in Multiple Myeloma and Have Strong Implications for Adverse Prognosis
}

\author{
Wei Wang ${ }^{a}$ Yuyue Ren ${ }^{b} \quad$ Lianjie Wang ${ }^{a} \quad$ Weiwei Zhao ${ }^{a} \quad$ Xiushuai Donga \\ Jiaqi Pan ${ }^{a}$ Haiyan Gao ${ }^{a}$ Yaoyao Tian ${ }^{a}$ \\ aDepartment of Hematology, the Second Affiliated Hospital of Harbin Medical University, Harbin, \\ ${ }^{b}$ Department of Hematology, the First Affiliated Hospital of Xinxiang Medical University, Xinxiang, China
}

\section{Key Words}

Soce $\cdot$ Stim 1 - Orai 1 - Tumor $・$ Multiple myeloma

\begin{abstract}
Background/Aims: Multiple myeloma (MM) is a plasma cell neoplasm which constitutes about $10 \%$ of all hematologic malignancies. Despite the development and application of novel agents, MM still undergoes an aggressive and incurable course in the vast majority of patients. $\mathrm{Ca}^{2+}$ is one of the critical regulators of cell migration. $\mathrm{Ca}^{2+}$ influx is essential for the migration of various types of cells including tumor cells. However, the role of store-operated calcium entry (SOC) channels, the only $\mathrm{Ca}^{2+}$ channels of non-excitable cells, has not yet been reported in MM cell survival. Methods: We evaluated the expression of Stim1 and Orai1 (two key regulators of SOC) in MM tissues and cell lines by immunohistochemical assay, quantitative real-time PCR assay and western blot. MM cell lines were pretreated with pharmacological blockers and siRNAs, and then MM cell proliferation, cell cycle arrest, and apoptosis were examined by FACS (flow cytometry) assay, and Annexin V-FITC/PI staining. The correlation between the expression of Stim1 (or Orai1) level and outcome in MM were assessed by using Progress Free Survival (PFS). Results: Stim1 and Orai1 were both abundantly expressed in MM tissue and MM cell lines. Inhibition of SOCE reduced MM cell viability, and induced cell cycle arrest and apoptosis. Stim1 or Orai1 silencing also reduced cell viability, caused cell apoptosis and cell cycle arrest in MM cell lines. Over-expression of Stim1/Orai1 in MM patients was closely associated with the clinical outcome of MM. Conclusion: The Stim1/Orai1-mediated signaling participates in the pathogenesis of $\mathrm{MM}$, which represents an attractive target for future therapeutic intervention.
\end{abstract}




\section{Introduction}

Multiple myeloma (MM) is a plasma cell neoplasm which constitutes about $10 \%$ of all hematologic malignancies, characterized by uncontrolled clonal proliferation of malignant plasma cells within the bone marrow, with associated monoclonal immunoglobulin and protein fragments in blood and urine. Novel agents are currently under development for the treatment of relapsed/refractory MM, including immunomodulatory drugs, proteasome inhibitors, monoclonal antibodies, cell signaling targeted therapies, and strategies targeting the tumor microenvironment [1-3]. The molecularly targeted therapies of MM are based on unique cell signaling pathways activated in $\mathrm{MM}$ and not in normal cells, including inhibitors of HDAC, PI3K/AKT/mTOR, p38 MAPK, Hsp90, Wnt, Notch and Hedgehog [4]. Despite promising results that have been recently obtained, the efficacy of these agents used alone is still limited and can be significantly enhanced by combination with traditional chemotherapeutic drugs. These latest therapies have led to an increase of patient survival time. Unfortunately, MM undergoes an aggressive and incurable course in the vast majority of patients. Although treatments have improved, nearly all MM patients relapse and die from progression of the disease [5]. Drugresistance remains a major clinical challenge for MM treatment. The main cause of resistance in MM is the minimal residual disease cells that are resistant to the original therapy such as bortezomib and high dose melphalan in stem cell transplant [6]. It is thus necessary and important to identify novel mechanisms that can inhibit proliferation and viability of MM cells and discover new targets to develop efficient tumor suppressing strategies.

In most types of cells, modulation of intracellular $\mathrm{Ca}^{2+}$ levels provides versatile and dynamic signaling that mediate various cellular processes, such as proliferation, migration, and gene expression [7]. $\mathrm{Ca}^{2+}$ is also one of the critical regulators of cell migration and the $\mathrm{Ca}^{2+}$ influx is essential for the migration of various cell types, including tumor cells $[7,8]$. In non-excitable cells, store-operated $\mathrm{Ca}^{2+}$ (SOC) channels in the plasma membrane are the major $\mathrm{Ca}^{2+}$ entry pathway [9]. Store-operated $\mathrm{Ca}^{2+}$ entry (SOCE), by definition, is activated by $\mathrm{Ca}^{2+}$ efflux from the internal store. Two genes, stromal-interaction molecule1 (Stim1) and the plasma membrane $\mathrm{Ca}^{2+}$ channel protein Orai1 (also known as CRACM1), are responsible for SOCE activation [9]. Stim1 is a single-pass transmembrane endoplasmic reticulum (ER) protein with multiple predicted protein interactions or signaling domains [10]. Orai1 is an essential component of $\mathrm{Ca}^{2+}$ release-activated $\mathrm{Ca}^{2+}$ channels, which are highly $\mathrm{Ca}^{2+}$-selective SOC channels in the plasma membrane. Stim 1 monitors $\mathrm{ER} \mathrm{Ca}^{2+}$ store concentration depletion and activates Orai1, allowing store-operated $\mathrm{Ca}^{2+}$ entry to occur [11]. When the $\mathrm{ER} \mathrm{Ca}^{2+}$ store is depleted by inositol 1, 4,5-triphosphate(IP3)-induced $\mathrm{Ca}^{2+}$ release or by inhibition of the ER $\mathrm{Ca}^{2+}$-ATPase pump, Stim1 unbinds $\mathrm{Ca}^{2+}$ from the $\mathrm{Ca}^{2+}$-binding EF hand motif near the amino terminus of Stim 1 in the ER lumen and translocates to the plasma membrane where it can interact with Orai1, resulting in an inward $\mathrm{Ca}^{2+}$ current into the cell [12]. The SOCE through the SOC channels thus provides $\mathrm{Ca}^{2+}$ for refilling $\mathrm{ER} \mathrm{Ca}^{2+}$ stores, as well as generates sustained $\mathrm{Ca}^{2+}$ signals that control essential functions such as gene expression, cell metabolism and exocytosis [9, 13]. Feske S et al [14]. demonstrated that Stim1 and Orai1 mRNA were widely expressed in different human tissues, and strongly presented in lymphoid cells. The identification of these two molecules greatly advanced our understanding of the molecular mechanisms of SOCE in various cellular systems including the immune system [15-17], hemostasis and thrombosis [18], skeletal and smooth muscle signaling [15, 19], and cancer biology [15]. In estrogen receptor negative breast cancer cells, SOCE is mediated by Stim1/ Orai1 proteins [20]. Recently, the treatment with a pharmacological inhibitor of SOCE and Stim1 siRNA decreased tumor metastasis in animal models of breast cancer [21]. Moreover, SOCE was confirmed as the main $\mathrm{Ca}^{2+}$ influx pathway involved in controlling proliferation of human hepatoma cell Stim1 or Orai1 knock down [22] and SOCE inhibitions have been shown to induce suppression of tumor cell proliferation by arresting cell cycle at G0/G1 or G2/M phase and inhibiting tumor cell metastasis in tumor cells such as leukemia [23], breast cancer [24], prostate cancer [25] and human cervical cancer [26].

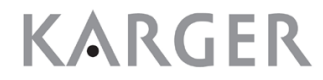


Although intensive studies have been carried out, little is known about the role of $\mathrm{Ca}^{2+}$ dysregulation or SOCE in MM. In this study, we evaluated the expression of Stim1 and Orai1 in MM tissues and cell lines, and examined the effect of SOCE inhibition on MM cell proliferation, cycle arrest, and apoptosis by using pharmacological blockers and siRNAs for Stim1 and Orai1. Moreover, we also evaluate if SOCE plays an important role in prognosis of MM.

\section{Materials and Methods}

\section{Cell culture}

KM3 and U266 (multiple myeloma cell line) were obtained from the Shanghai biological institute of Chinese academy of sciences cell bank (Shanghai, China). U266 were cultured in RPMI 1640 medium supplemented with $10 \%$ fetal calf serum (FCS), and KM3 were cultured in DMEM medium supplemented with $10 \%$ fetal calf serum (FCS).Cells were maintained at $37^{\circ} \mathrm{C}$ in a humid atmosphere of $5 \% \mathrm{CO}_{2}$ in air. The cells were counted each day with a hemacytometer and then passaged every 2 days to maintain them in the exponential growth phase (i.e. maintained at $1-2 \times 10^{5}$ cells $/ \mathrm{ml}$ ). To evaluate cell viability, cells were mixed with equal volumes of $0.4 \%$ trypan blue solution, and then immediately examined under the microscope. All of the cell culture reagents were purchased from Sigma Company.

\section{Clinical research specimens and immunohistochemistry}

This study enrolled $60 \mathrm{MM}$ patients and 25 control individuals including 8 iron-deficiency anemia patients, 8 megaloblastic anemia patients, 5 healthy people(negative control) and 4 cervical carcinoma patients (positive control) from the second affiliated hospital of Harbin Medical University (Harbin, China). The median age of 60 Chinese MM patients (36 males, 24 females) was 59.3 years old (range 41-79). According to the Durie-Salmon (DS) staging system, 7 patients were stage I, 5 patients stage II, and the remaining 48 patients stage III. Bortezomib combined with dexamethasone administration (BD regimen) was evaluated in patients with multiple myeloma. Bortezomib $1.3 \mathrm{mg} / \mathrm{m}^{2}$ subcutaneously or intravenously days 1, 4, 8 and 11; and dexamethasone $20 \mathrm{mg}$ days $1,2,4,5,8,9,11$ and 12 once every 21 days was given for up to 2-8 cycles. All tissue samples were embedded in paraffin. Monoclonal mouse anti-Stim 1 or anti-Orai1 antibody (Abcam) was used in the Stim1 or Orai1 immunohistochemistry assay of these samples. Primary antibodies to Orai1 (P20, goat polyclonal IgG, SC-74778) and to Stim1 (H-180, rabbit polyclonal IgG, SC68897) were purchased from Santa Cruz Biotechnology (Santa Cruz, CA). Compatible Alexa Fluor ${ }^{\circledR}$ labeled secondary antibodies were purchased from Invitrogen (Carlsbad, CA).

\section{Quantitative real-time PCR}

To quantify mRNA expression of Stim1/Orai1, total RNAs were extracted from human bone marrow tissue specimens and cell lines with TRIzol reagent (Takara). The isolated total RNA was reverse transcribed and used in a two-step quantitative real-time PCR according to the manufacturer instructions. The following primer sets were used: Stim1 (Accession No.NM_003156): 5'-AGG GCA TCT TGC CTG GAG A-3' (forward), 5'-CAC CTC ATC CAC GGT CCA A-3' (reverse); Orai1 (CRACM1) (Accession No.NM_032790): 5'-CCA TGG TGG CAA TGG TGG A-3' (forward), 5'CTC CTG GAA CTG TCG GTC A-3' (reverse); $\beta$-actin, 5'-AGC GGG AAA TCG TGC GTG-3 (forward), 5-CAG GGT ACA TGG TGG TGC C-3' (reverse). PCR products were separated by $2.0 \%$ agarose gel electrophoresis and visualized by GelRedTM (Biotium, Inc., Hayward, CA) staining.

\section{Western blot}

Western blotting was performed to confirm the expression of Stim1 and Orai1. The membrane was blocked for nonspecific binding by incubation with $5 \%$ fat-free dry milk in TBST $(10 \mathrm{mM}$ Tris $\cdot \mathrm{HCl}, \mathrm{pH}$ 7.4, $150 \mathrm{mM} \mathrm{NaCl}$, and $0.1 \%$ Tween 20) for $60 \mathrm{~min}$. Mouse monoclonal anti-human Stim1 (1:1000; Cat. No. 610954, BD Pharmingen, Franklin Lakes, NJ) and rabbit anti-Orai1 (1:1000; Cat. No. 08264, Sigma) antibodies were used as the primary antibodies, and the antibodies was diluted in blocking buffer and incubated with the membrane overnight at $4^{\circ} \mathrm{C}$. The membrane was washed in TBST (10 min, 5 times), 


\section{Cellular Physiology Cell Physiol Biochem 2018;48:2273-2285 \begin{tabular}{ll|l} 
and Biochemistry Published online: 15 August, 2018 & $\begin{array}{l}\text { (c) } 2018 \text { The Author(s). Published by S. Karger AG, Basel } \\
\text { www.karger.com/cpb }\end{array}$
\end{tabular}}

Wang et al.: Orai1 and Stim1 Expression and SOCE in MM

incubated with secondary antibody for $60 \mathrm{~min}$ at room temperature, and washed again in TBST (10 min, 5 times), and the immunoreactive bands were visualized using ECL plus reagent (Amersham Biosciences, Piscataway, NJ).

\section{MACS MicroBeads}

Flow cytometry was used to measured MACSs MicroBeads labelled CD138 cells, MM cell lines (KM3 and U266) and bone marrow mononuclear cells of MM patients and healthy people. Cells were incubated with human FcR Blocking Reagent (Miltenyi Biotec), CD138 antibody (Miltenyi Biotec), 7-AminoActinomycin (7-AAD) staining solution (Becton Dickinson, Heidelberg, Germany) and Labeling Check Reagent-FITC and measured using flow cytometer (BD Bioscience).

\section{Trypan blue assay}

Cells $\left(10 \times 10^{3}\right.$ cells/well $)$ were seeded into 48-well plates and treated with various doses of SKF-96365 (Sigma Aldrich), DES (Sigma Aldrich) or 2-APB (Sigma Aldrich) for 48h. At the end of the incubation period, cell viability was assessed by trypan blue assay. Cells were mixed with the same volume of $0.4 \%$ trypan blue solution and immediately examined under the microscope. Inhibition graphs were plotted with mean values obtained from each concentration relative to control values, and the half maximal inhibitory concentration (IC50) were calculated by SPSS.

\section{Cell cycle analysis}

Cells $\left(4 \times 10^{5}\right.$ cells/well) were seeded in 6-well plates and treated with various doses of SKF-96365, DES or 2-APB for 48 hours. The cells were harvested, and washed with phosphate-buffered saline (PBS), and then fixed with $70 \%$ ethanol at $-20^{\circ} \mathrm{C}$ overnight. After an additional washing, cells were incubated with RNase A (20 $\mu \mathrm{g} / \mathrm{mL})$ at $37^{\circ} \mathrm{C}$ for $30 \mathrm{~min}$, stained with propidium iodide (100 $\mu \mathrm{g} / \mathrm{mL}$; Sigma Aldrich) for 10 min, and analyzed with flow cytometry (BD FACSC auto TM II).

\section{Annexin-V Fluorescein Isothiocyanate/Propidium Iodide (FITC/PI) Double Staining Assay}

Annexin V-FITC apoptosis detection kit (BD Pharmingen, San Diego, CA) was used to determine the percentage of dead, viable, apoptotic and necrotic cells after treatment with drugs according to the manufacturer's instructions. The cells were seeded at $4 \times 10^{5}$ cells/well in 6 -well plates. After treatment with drugs, the cells were harvested, washed twice with ice-cold phosphate-buffered saline (PBS) and resuspended in $100 \mu \mathrm{l}$ of binding buffer. A total of $5 \mu \mathrm{l}$ of Annexin V-FITC and $10 \mu \mathrm{l}$ of PI were added and the mixture was incubated in dark for $30 \mathrm{~min}$. Then, $400 \mu \mathrm{l}$ of binding buffer was added to the cells. The labeled cells $(10,000$ /sample) were analyzed by measuring fluorescence intensity using flow cytometer (BD Bioscience) in conjunction with Cell Quest software (BD Biosciences, San Jose, CA).

\section{DAPI assay}

Apoptotic death was evaluated by nuclear fragmentation in 46-diamidino-2-phenylindole-2 $\mathrm{HCl}$ (DAPI)-staining cells. After fixation in $70 \%$ ethanol for $10 \mathrm{~min}$, cells were stained with DAPI $(0.5 \mu \mathrm{g} / \mathrm{ml})$ for $10 \mathrm{~min}$, and observed under UV-light using a confocal microscope (R2100AG2, Bio-Rad, Melville, NY). Ten different fields were randomly selected for counting 300 cells. The percentage of cells with fragmented nuclei was then calculated.

\section{Transmission electron microscopy (TEM)}

The ultrastructural morphology of the apoptotic death cells were further analyzed by transmission electron microscopy (TEM, JEM-1011, Japan). The mean size, size distribution and zeta potential of MM cells were analyzed by using a Malvern mastersizer 2000 (Malvern, UK).

\section{Confocal Microscopy}

Cells were detached from culture flasks by 2-3 minutes incubation with 2\% EDTA in PBS. Detached cells were washed twice with fresh culture medium and incubated in $2 \mu \mathrm{M}$ Fura- $2 \mathrm{AM}$ in medium at room temperature for $30 \mathrm{~min}$. After one wash with PBS, cells were plated on a No.1 glass cover slip mounted in a chamber on the stage of an inverted microscope (Nikon TE2000; Tokyo, Japan). A Nikon 40×UV oil-immersion objective lens was used to visualize the cells. Cells were illuminated by alternating excitation at 340 and 
$380 \mathrm{~nm}$ wave-lengths every 10s; rapid filter changes for ratiometric imaging were computer controlled via a Lambda 10-2 filter wheel and changer and MetaFluor 6.3 software (Molecular Devices, Sunnyvale, CA); fluorescence images were acquired using a CCD camera (Micro Max RTE CCD-728, Photometrics; Tucson $\mathrm{AZ}$ ). Images were corrected for background fluorescence and shade using functions in MetaFluor and were recorded. Averages of fluorescence intensity ratio at $340 / 380 \mathrm{~nm}$ in regions of interest defined in individual cells of each stored image provided an estimate of intracellular calcium concentration, which were analyzed in Excel.

\section{SiRNA knockdown of Orai1 and Stim1}

SiRNA and transfection reagents were purchase from Qiagen (Valencia,CA). A reverse protocol was performed according to manufacturer's instructions. Specifically, a mixture of $0.5-1 \mu \mathrm{L}$ siRNA stock solution (10-20 $\mu \mathrm{M}$ in RNase free water) $/ 1.5 \mu \mathrm{L}$ Hiperfect transfection reagent/100 $\mu \mathrm{L}$ serum free medium was applied into each well of a 96-well culture plate. The mixtures were incubate for 5 to 10 min to allow for the formation of transfection complexes, then an additional 1000 cells in $100 \mu \mathrm{L}$ complete culture medium were added to each well for transfection. Calculated final siRNA concentrations were 50nM. Cells were incubated in $5 \% \mathrm{CO}_{2}$ at $37^{\circ} \mathrm{C}$ for $32-72 \mathrm{~h}$. MTS cell proliferation assay was then performed. To measure $\mathrm{Ca}^{2+}$ concentration, siRNA treatment was performed in cells cultured on a $35 \mathrm{~mm}$ dish with a cover glass at the bottom $(0.5 \mathrm{~cm}$ diameter). Two different sequences of Orai1 and Stim 1 siRNAs were used for protein knockdown. The oligonucleotide sequences of RNA duplexes for siRNA assays were: Orai1, forward GGAUCAUGA CUA CCC GCCATT/reverseUGGCGGGUAGUCAUGAUCCGT; Stim1, GCCACG UCU UCC AAUGGUATT/UACCAUUGGAAGACGUGGCAT; Scramble sequences were used for control siRNA treatment.

\section{Statistical analysis}

Statistical analysis was performed using the software package SPSS version 20.0 and GraphPad Prism 5. Survival curves were constructed with the use of Kaplan-Meier estimates. All p values were two sided. Differences with $\mathrm{p}$ values of less than 0.05 are considered significant.

\section{Results}

\section{Increased expression of Stim1 and Orai1 in MM tissues and cell lines}

We firstly evaluated the expression Stim1/Orail1 in bone marrow's samples of 60 MM patients and 21 negative controls ( 8 iron deficiency anemia patients, 8 megaloblastic anemia patients, and 5 healthy individuals) , and pathological tissues of 4 cervical carcinoma patients were used as positive controls [26] by immunohistochemistry staining. The data showed that Stim 1 and Orai1 protein were highly expressed in MM bone marrow tissues and cervical cancer tissues (positive control), while only low levels were detected in the MA bone marrow tissues (Fig. 1A).

We then examined the expression levels of Stim1 and Orai1 in MM cell lines KM3 and U266 by RT-PCR assay. In accordance with human tissue samples, the mRNA levels of Stim1 and Orai1 were higher in primary MM cells, KM3 and U266 cells than healthy controls (Fig. 1B).

We then examined mRNA levels of Stim1 and Orai1 in 60 bone marrow (BM) samples from MM patient and 21 from negative group (8 MA, 8 IDA and 5 healthy controls) by quantitative real-time PCR. The mRNA levels of Stim 1 and Orai 1 were higher in MM tissue than negative controls (Fig. 1C).

Furthermore, we evaluated the expression of Stim1 and Orail1 in MM cell lines and MM patients' samples by Western Blot. As shown in Fig 1D and E, the expression levels of Stim1 and Orail1 were higher in KM3, U266 cells and MM patients' samples than negative controls.

The viability of human MM cell lines can be inhibited by SOCE inhibitors

To decipher the role of up-regulated SOC (Stim1 and Orai1) in human MM, we examined the effect of three inhibitors of SOCE (SKF-96365, DES, and 2-APB) on the viability of MM cell lines and primary multiple myeloma cells. The inhibitors of SOCE (SKF-96365, DES, and 
2-APB) suppressed the viability of all these cells in a dose-dependent manner (Fig. 2A-C). The IC50 of SKF-96365 was $2.38 \pm 0.35 \mu \mathrm{M}$ (KM3 cell line), $3.06 \pm 0.51 \mu \mathrm{M}$ (U266 cell line) and $3.35 \pm 0.58 \mu \mathrm{M}$ (primary multiple myeloma cells). The IC50 of DES was $23.65 \pm 3.42 \mu \mathrm{M}$ (KM3 cell line), $29.87 \pm 2.91 \mu \mathrm{M}$ (U266 cell line) and $27.24 \pm 3.54 \mu \mathrm{M}$ (primary multiple myeloma cells). The IC50 of 2 -APB is $32.37 \pm 3,74 \mu \mathrm{M}$ (KM3), 33.69 $\pm 4.01 \mu \mathrm{M}$ (U266) and $39.28 \pm 4.45 \mu \mathrm{M}$ (primary multiple myeloma cells).

Fig. 1. Expression of Stim1and Orai1 in MM bone marrow tissues and MM cell lines. (A) Immunohistochemical staining of Stim1 and Orai1 in paraffin embedded tissue sections from cervical cancer tissue (positive control), MM bone marrow tissue and MA bone marrow tissue (negative control). Images were photographed at $40 \times$ magnification. (B) The mRNA expression of Stim1 and Orai1 in the bone marrow mononuclear cells of healthy individual, primary MM cells and MM cell lines detected by RT-PCR. (C) The mRNA levels of Stim1 and Orai1 in 60 cases of BM samples in MM patient and 21 cases of BM samples in negative group (8 iron-deficiency anemia patients, 8 megaloblastic anemia patients and 5 healthy individuals) were analyzed by quantitative real-time PCR. (D) Representative western blot showing protein levels of Stim 1 and Orai1 in 2 cases
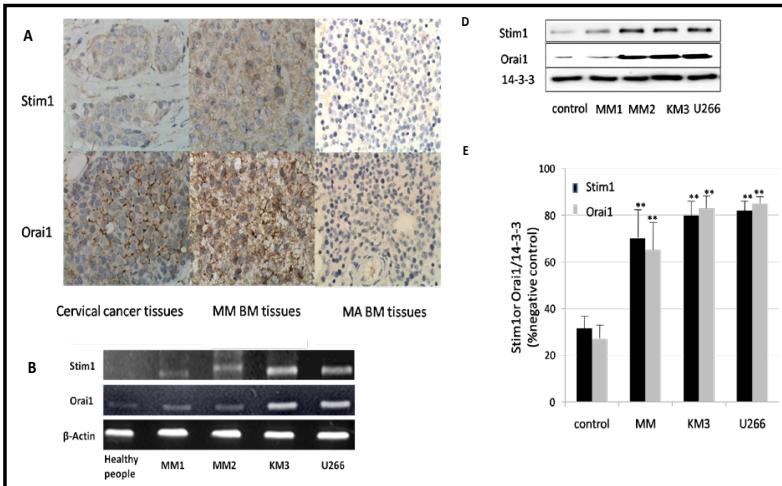
of MM patients and MM cell lines.(E) Mean

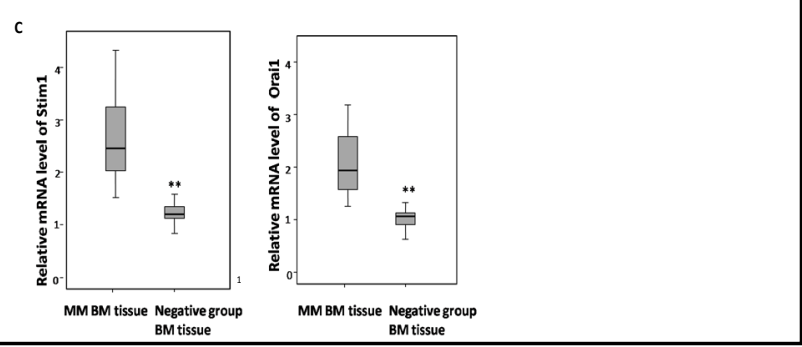
intensity of Stim1 and Orai1 bands relative to 14-3-3 in 60 cases of BM samples from MM patients and 21 cases of BM samples from negative group (8 iron-deficiency anemia patients, 8 megaloblastic anemia patients and 5 healthy people). All values were presented as mean \pm SD of three independent experiments. $* * \mathrm{P}<0.01$ vs. negative group.

Fig. 2. Calcium entry blockers inhibited the viability of human MM cell lines and primary multiple myeloma cells. (A-C) KM3, U266 cells and primary multiple myeloma cells were treated with SKF-96365, DES and 2-APB for 48 hours. (D-F) Cell viability was measured by trypan blue exclusion assay. The viability of KM3, U266 cells and primary multiple myeloma cells treated with SKF-96365 $(5 \mu \mathrm{M})$, DES $(30 \mu \mathrm{M})$ and 2 -APB $(40 \mu \mathrm{M})$ respectively for $24 \mathrm{~h}$ and $48 \mathrm{~h}$ detected by trypan blue exclusion assay. Values for viability were expressed as percentage of total cells. Data are presented as mean \pm SD of 3 separate experiments. ${ }^{*} \mathrm{p}<0.05$ vs control.

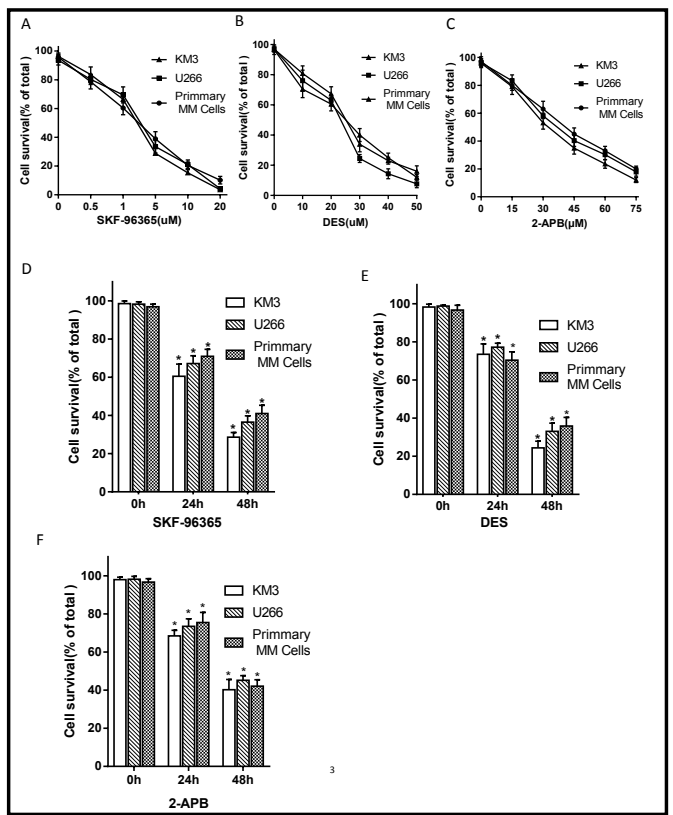


Trypan blue assay shows that after treatment of KM3, U266 and primary multiple myeloma cells with SKF-96365 $(5 \mu \mathrm{M})$, DES $(30 \mu \mathrm{M})$ and 2-APB $(40 \mu \mathrm{M})$, the viability started to decrease at $24 \mathrm{~h}$ and further reduced in a time-dependent manner (Fig. 2D-F). These data indicated that blocking of SOCE produced both dose-dependent and time-dependent inhibitory effect on the viability of human MM cells.

\section{SOCE blockers caused MM cell cycle arrest}

We observed the influence of SOCE on MM cell cycle arrest in an FACS (flow cytometry) assay. The data revealed that calcium entry blockers (SKF-96365 $5 \mu \mathrm{M}$, DES $30 \mu \mathrm{M}$ or 2-APB $40 \mu \mathrm{M}$ ) significantly increased the proportion of cells at G2/M phases of cell cycle after 48h's treatment compared to vehicle controls $(\mathrm{P}<0.05)$. The increase in the $\mathrm{G} 2 / \mathrm{M}$ cell population was accompanied by a concomitant decrease in the cell population of $S$ and G0/G1 phases (Fig. 3A-C).

\section{Calcium entry blockers induced MM cell apoptosis}

The MM cell lines KM3 and U266 were incubated with the calcium entry blockers (SKF$963655 \mu \mathrm{M}$, DES $30 \mu \mathrm{M}$ or 2 -APB $40 \mu \mathrm{M}$ ) for $48 \mathrm{~h}$, respectively. Annexin V-FITC/PI staining was used to evaluate cell apoptosis. These data indicated that SKF-96365, DES and 2-APB treatment significantly increased apoptotic cell numbers in MM cell lines as shown in Fig. $4 \mathrm{~A}-\mathrm{D}(\mathrm{P}<0.05$ vs. Control).

Fig. 3. Calcium entry blockers induced MM cell cycle arrest. KM3 and U266 cells were treated with SKF$96365(5 \mu \mathrm{M}, \mathrm{A}), \mathrm{DES}(30 \mu \mathrm{M}$, B) and 2-APB $(40 \mu \mathrm{M}, \mathrm{C})$ for $48 \mathrm{~h}$, respectively. Data are presented as means \pm SD of three independent

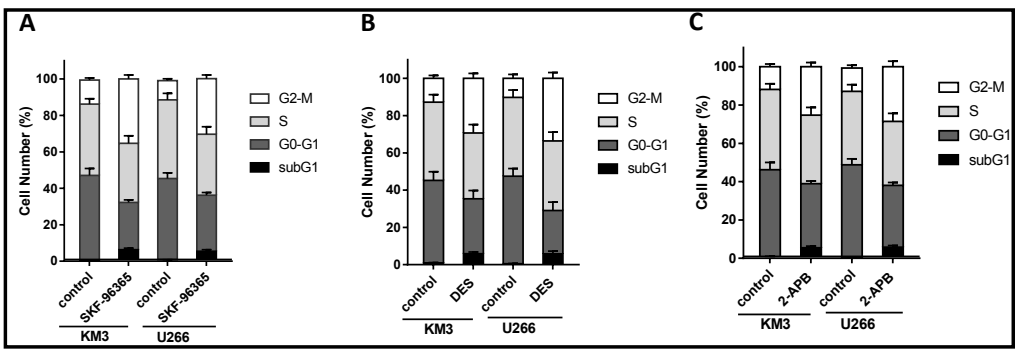
experiments.

Fig. 4. Calcium entry blockers induced MM cell apoptosis in culture. (A) Apoptotic death of KM3 and U266 cells treated with SKF-96365 $(5 \mu \mathrm{M})$, DES $(30 \mu \mathrm{M})$ and 2 -APB $(40 \mu \mathrm{M})$, respectively for $48 \mathrm{~h}$ detected by flow cytometry after Annexin V-FITC/PI staining. (B-D) Statistical analysis of the apoptosis of KM3 and U266 after SKF-96365 $(5 \mu \mathrm{M}), \mathrm{DES}(30 \mu \mathrm{M})$ and 2 -APB $(40 \mu \mathrm{M})$ treatment with V-FITC/PI double staining. (E) Apoptotic death of KM3 and U266 cells treated with SKF$96365(5 \mu \mathrm{M})$, DES $(30 \mu \mathrm{M})$ and 2 -APB $(40 \mu \mathrm{M})$ for $48 \mathrm{~h}$ examined by electron microscopy. Data are expressed as mean \pm SEM. $n=3$ individual experiments. ${ }^{*} \mathrm{p}<0.05$ vs control.

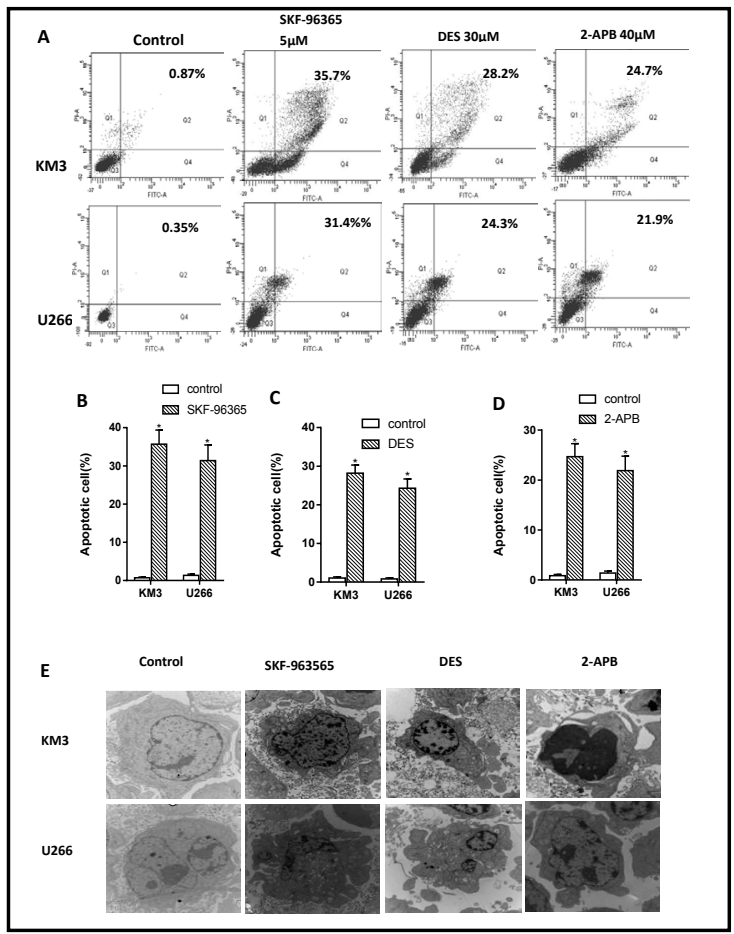


Fig. 5. Calcium entry blockers restrained extracellular calcium influx via the channel of SOCE. Tharpsigargin (TP, $1 \mu \mathrm{M}$, marked by filled bar) applied to cells in 0 $\mathrm{Ca}^{2+}$ solution for $400 \mathrm{sec}$ to deplete internal $\mathrm{Ca}^{2+}$ store, followed by reintroduction of $2 \mathrm{mM} \mathrm{Ca}^{2+}$ solution, induced stable increases in $\mathrm{Ca}^{2+}$ signal, a characteristic SOCE $(340 / 380$ fluorescence ratio from 20 cells. Data shown is mean \pm SEM. A: SKF-96365 applied in the solution induced inhibition of $\mathrm{Ca}^{2+}$ signal at $30 \mu \mathrm{M}$ (marked by arrow); the subsequent $0 \mathrm{Ca}^{2+}$ solution ( $\mathrm{Ca} 0)$ washing abolished $\mathrm{Ca}^{2+}$ signal to baseline. $\mathrm{B}$ and C shows similar experiments to A. Diethylstilbestrol (DES, $10 \mu \mathrm{M}$, marked by the arrow) and 2 -APB $(40 \mu \mathrm{M}$, marked by the arrow) applied in $\mathrm{Ca}^{2+}$ solution abolished

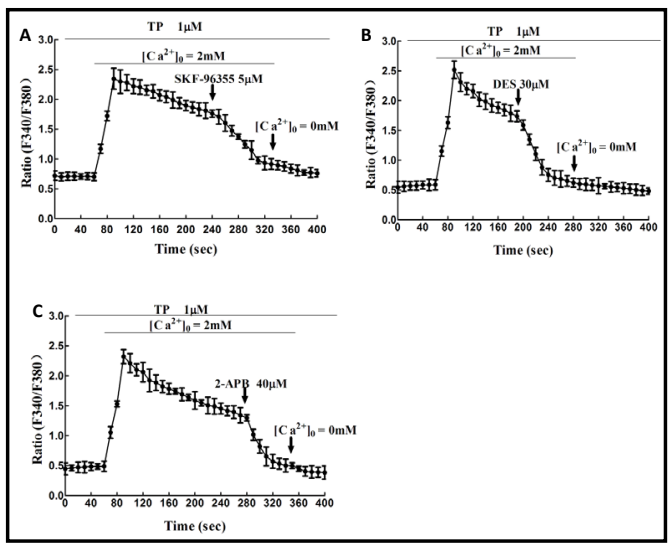
the large portion of $\mathrm{Ca}^{2+}$ entry signals.

The ultrastructural morphology of the MM cells analyzed by transmission electron microscopy (TEM) showed that calcium entry blockers induced typical nuclear features of apoptosis including the pycnosis of cell nucleus and the appearance of apoptotic body with nuclear membrane (Fig. 4E).

\section{Calcium entry blockers induced [ $\left.\mathrm{Ca}^{2+}\right]$ i reduction in KM3 cells}

To identify the relationship between calcium entry blockers and the intracellular $\mathrm{Ca}^{2+}$ concentration, thapsigargin (TP), the endoplasmic reticulum $\mathrm{Ca}^{2+}$-ATPase blockers, was used to deplete intracellular calcium stores in a $\mathrm{Ca}^{2+}$ free solution. Subsequent sustained increase in intracellular $\mathrm{Ca}^{2+}$ from baseline and characteristic SOCE was induced after the reintroduction of $\mathrm{Ca}^{2+}$ in the external solution. We conducted $\mathrm{Ca}^{2+}$ imaging for $400 \mathrm{sec}$ on KM3 cells in order to distinguish possible influence of SKF-96365 on homeostasis. As shown in Fig. 5A, SKF-96365 abolished the large portion of the $\mathrm{Ca}^{2+}$ entry signals. We also found that others calcium entry blockers DES and 2-APB showed the same effect as SKF-96365 on [Ca $\left.{ }^{2+}\right]$ i (Fig. 5B and C).

\section{SOCE is essential for MM cell proliferation}

MM cell lines KM3 and U266 cells were pretreated with the siRNAs of Stim1 or Orai1 for $48 \mathrm{~h}$, and the efficiency of Stim1/Orai1 knock down was validated by western blot (Fig. 6A and $\mathrm{B}$ ). Then the cellular $\mathrm{Ca}^{2+}$ stores were depleted by using TP in $\mathrm{Ca}^{2+}$ free solution and the SOCE was triggered by the reintroduction of $2 \mathrm{mM}$ external $\mathrm{Ca}^{2+}$. Fura-2 fluorescence calcium imaging was used to detect intracellular $\mathrm{Ca}^{2+}$ concentration changes. TP induced a transient peak of $\mathrm{Ca}^{2+}$ release from internal stores in a $\mathrm{Ca}^{2+}$ free external solution in the cells treated with Stim 1 or Orai1 siRNA, but a notably smaller $\mathrm{Ca}^{2+}$ entry in $2 \mathrm{mM}$ external $\mathrm{Ca}^{2+}$ solution. Both siRNAs of Stim1 and Orai1 induced significant decrease in $\mathrm{Ca}^{2+}$ entry in KM3 cells compared to the control. There was no significant difference in $\mathrm{Ca}^{2+}$ entry between Stim1 and Orai1 siRNA treated cells (Fig. 6C).

Then we examined the effect of Stim1 or Orai1 silencing on cell apoptosis by TEM. Knockdown of Stim1 or Orai1 caused typical nuclear features of apoptosis including the pycnosis of cell nucleus and the appearance of apoptotic body with nuclear membrane (Fig. 6D). No apoptotic changes were observed in control siRNA. KM3 cells were treated with vehicle, control siRNA, Stim 1 or Orai1 siRNA for $48 \mathrm{~h}$ and then cell viability was measured by trypan blue exclusion assay. It showed that knock-down of Stim1 or Orai1 inhibited MM cell viability (Fig. $6 \mathrm{E},{ }^{* *} \mathrm{p}<0.01$ vs control). Subsequently, we quantitatively analyzed apoptosis induced by silencing of Stim 1 or Orai1 in KM3 and U266 cells by Annexin V-FITC/PI staining. We found that apoptotic ratio in Stim1 or Orai1 siRNA groups were higher than control groups (Fig. 6F and G). 


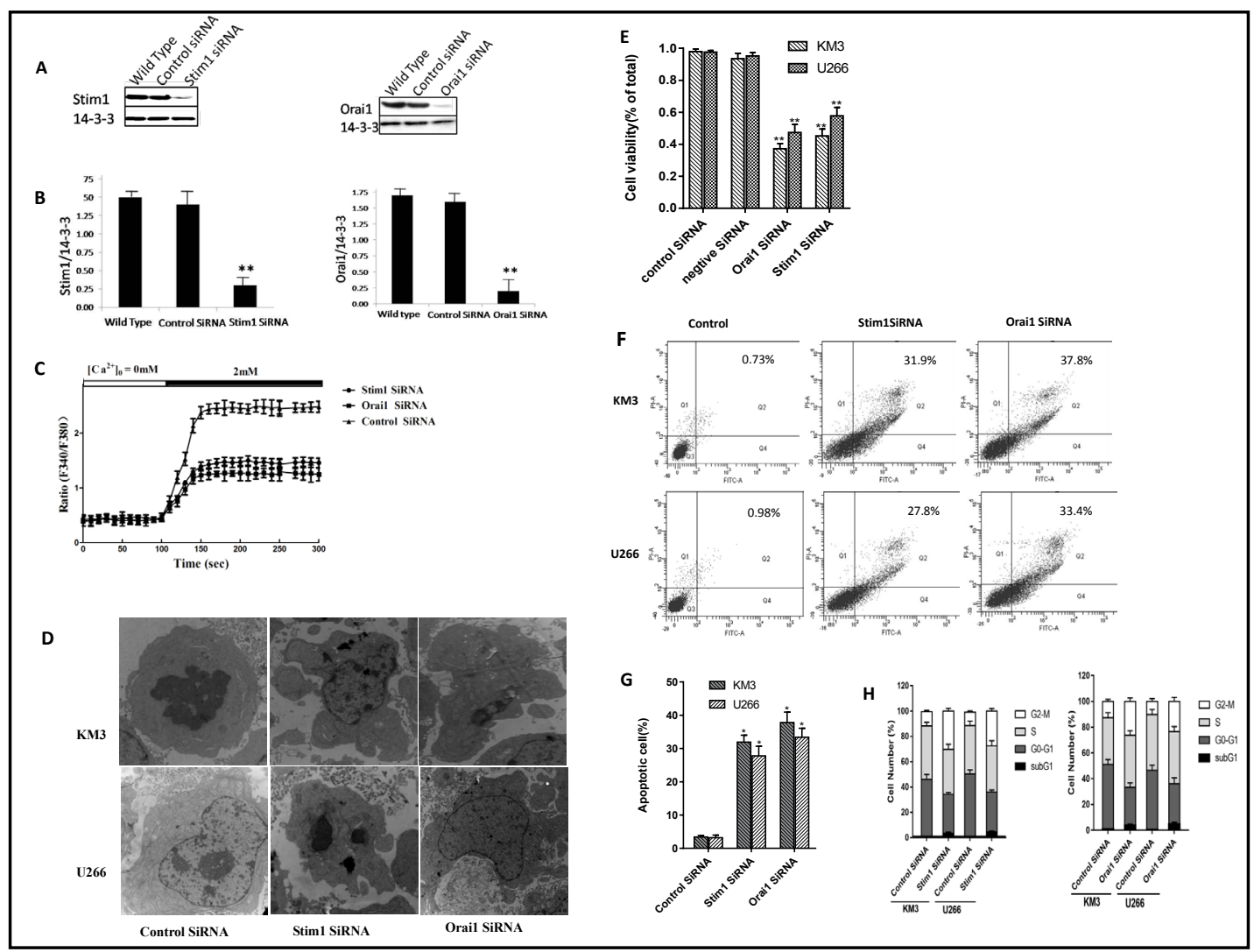

Fig. 6. Knockdown of Stim1 or Orai1 by siRNA on calcium homeostasis and proliferation, apoptosis, and cell cycle of MM cells. (A) Stim1 and Orai1 knockdown by siRNA in MM KM3 cell lines. (B) Stim1 and Orai1 proteins levels normalized to $14-3-3$ protein ( $\%$ of negative control, ${ }^{* *} \mathrm{p}<0.01$ vs. negative control). (C) Stim1 or Orai1 siRNA inhibits SOCE of KM3 cells. To measure $\mathrm{Ca}^{2+}$ entry, KM3 cells loaded with Fura-2/AM(2 $\left.\mu \mathrm{M}\right)$ were pre-incubated in $\mathrm{Ca}^{2+}$-free media plus $2 \mu \mathrm{M}$ thapsigargin for $30 \mathrm{~min}$ to deplete the internal $\mathrm{Ca}^{2+}$ store. Each trace was averaged from at least 20 single cells. (D) Apoptotic death of KM3 cells treated with Stim1 and Orai1 siRNA observed by using electron microscopy. (E) Knock-down of Stim1 or Orai1 inhibited MM cell viability. KM3 cells were treated with vehicle, control siRNA, Stim1 or Orai1 siRNA for 48h and then measured by trypan blue exclusion assay. ${ }^{* *} \mathrm{p}<0.01$ vs control. (F) Apoptotic death of KM3 cells treated with Stim1 or Orai1 siRNA for $48 \mathrm{~h}$ detected by flow cytometry after Annexin V-FITC/PI double staining. (G) Statistical analysis of the apoptosis of KM3 after Stim1 or Orai1 siRNA treatment with V-FITC/PI double staining. Data are expressed as mean \pm SEM. $n=3$ individual experiments. ${ }^{*} p<0.05$ vs control. (H) Knock-down of Stim1 or Orai1 induced MM cell cycle arrest. KM3 treated with Stim1 or Orai1 siRNA for 48h. Data are presented as means \pm SD of three independent experiments.

Fig. 7. The expression level of Stim1/Orai1 is associated with the clinical outcome of MM. (A) The expression levels of Stim 1 and Orai1 mRNA in different disease stage groups assessed by quantitative real-time PCR. ${ }^{* *} \mathrm{p}<0.01$ vs. control (B) Western blot results of Stim1 and Orail1 expression levels in different disease stage groups. ${ }^{* *} \mathrm{p}<0.01$ vs. negative control. (C) Survival analysis of MM patients according to Stim1/Orail1 expression levels.

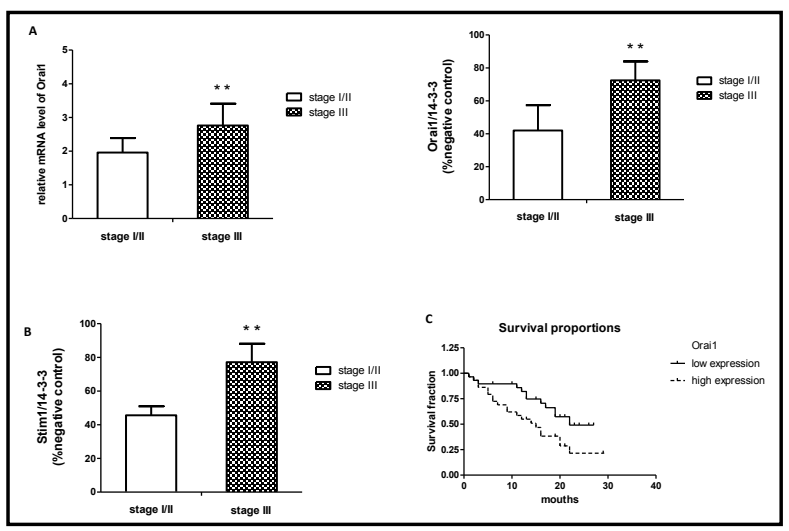


We also examined the effect of Stim1 or Orai1 silencing on cell cycle arrest in MM cells. As shown in Fig. $6 \mathrm{H}$, compared to control siRNA groups, Stim1 or Orai1 siRNA groups significantly increased the proportion of cells at $G 2 / \mathrm{M}$ phases of cell cycle $(\mathrm{P}<0.05)$. The increase in the $\mathrm{G} 2 / \mathrm{M}$ cell population was accompanied by a concomitant decrease in the population of $S$ and G0/G1 phases. These data suggested that Stim1 or Orai1 silencing caused cell apoptosis and cell cycle arrest in MM cell lines.

The expression level of Stim1/Orai1 is associated with the clinical outcome of MM

We've evaluated the expression level of Stim1/Orail1 by quantitative real-time PCR in $60 \mathrm{MM}$ patient samples and found that the expression levels of Stim1/Orail1 in the stage III group was obviously higher than the stage II and I group (Fig. 7A). see the protein levels of Stim1 and Orail1 in stage II and I group was lower than the stage III group (Fig. 7B).

We divided the patients into two groups according to the expression level of Stim1. We assessed whether a direct correlation exists between Stim1 expression and clinical outcome in MM cases by using PFS as our clinical criterion (median follow-up 14.12 months). The median duration of PFS in patients with low Stim1 expression group was 15.33 months. However, the median duration of PFS in high Stim1 expression group was 13.34 months. These results indicated that high expression level of Stim1/Orail1 was associated with shorter PFS $(\mathrm{p}<0.05)$ (Fig. 7C).

\section{Discussion}

This study highlights the novel role of SOCE and channel components Stim1 and Orai1 in tumor malignant behavior. We showed that SOCE are important for MM cell proliferation, cell cycle arrest, and apoptosis. This conclusion is supported by the following evidences: (i) we found that bone marrow tissues of MM patients and MM cell lines (KM3, U266) highlyexpressed SOCE channel components Stim1 and Orai1. (ii) Stim1/Orai1-silencing in MM cell lines (KM3, U266) significantly inhibied cell proliferationand caused cell cyclearrest at the G2/M phases. We also observed the same effects by inhibitors of SOCE (SKF96365, 2-APB, and DES). (iii) Cell apoptosis was dramatically induced by Stim1/Orai1-silencing or inhibitors of SOCE (SKF96365, 2-APB, and DES). (iv) Stim1/Orai1-silencing or inhibitors of SOCE (SKF96365, 2-APB, and DES) inhibited SOCE of MM cells by decreasing store-operated calcium entry. (v) Highly-expression of Stim1/Orail1 was associated with shorter PFS in MM patients.

$\mathrm{Ca}^{2+}$ is a critical second messenger for a wide range of physiological and pathological processes. Elevation of intracellular $\mathrm{Ca}^{2+}$ levels may result from the entry of extracellular $\mathrm{Ca}^{2+}$ as well as the release of sequestered $\mathrm{Ca}^{2+}$ from intracellular stores such as the ER [27, 28]. In non-excitable cells, SOCE is the major pathway for $\mathrm{Ca}^{2+}$ entry $[29,30]$. SOCE are plasma membrane $\mathrm{Ca}^{2+}$ channels activated by a decrease in $\mathrm{Ca}^{2+}$ content in the ER [31]. Under physiological conditions, the activation of G-protein or tyrosine kinase-coupled receptors by binding a hormone or other agonist leads to the activation of PLC $\beta$ or PLC $\gamma$ and the formation of IP3. The combination of $\mathrm{IP}_{3}$ and $\mathrm{IP}_{3} \mathrm{R}$ then releases $\mathrm{Ca}^{2+}$ from the ER, finally activating SOCE. Similarly, in the current study, the addition of thepharmacological inhibition of the calcium pumps inhibitor thapsigargin (TP) in the absence of extracellular calcium causes the depletion of stored calcium in MM cell line KM3. Readdition of $\mathrm{Ca}^{2+}$ to the extracellular media, causes an influx of $\mathrm{Ca}^{2+}$ through the influx pathway responsible for $\mathrm{Ca}^{2+}$ store refilling, which as shown here can be blocked by inhibition of one of the molecular components of this $\mathrm{Ca}^{2+}$ influx pathway known as Stim1 or Orai1. This study found that inhibitors of SOCE could significantly reduce the $\left[\mathrm{Ca}^{2+}\right] \mathrm{i}$ in MM cells, which suggests that SOCE may be the defined $\mathrm{Ca}^{2+}$ channel mentioned previously.

MM is a malignancy of secretory plasma cells, and as such, they contain a highly developed endoplasmic reticulum (ER), characteristic of secretory cells. Landowski et al [32]. demonstrated that the dysregulation of $\mathrm{Ca}^{2+}$ homeostasis could lead to $\mathrm{MM}$ cell apoptosis, 
and alterations in $\mathrm{ER} \mathrm{Ca}^{2+}$ storage or release played a role in the observed dysregulation of $\mathrm{Ca}^{2+}$ homeostasis. However, the role of SOCE channels which act as the only $\mathrm{Ca}^{2+}$ channels of non-excitable cells in MM cell survival has not yet been reported. In this study, we showed that the protein expression level of Stim1 and Orai1 is higher in MM tissues than normal controls. These results are consistent with the up-regulation of SOCE components in tumor tissues [33]. This prompted us to hypothesize that $\mathrm{Ca}^{2+}$ entry via SOCE channels play important roles in sustaining MM cell proliferation and apoptosis. We speculate that the high abundance of Stim1 and Orai1 indicates the high risk of occurrence and metastasis of MM.

The critical question is whether Stim1/Orai1 contributes to the cancerous properties of MM cells. Emerging literature points to major role of Stim1/Orai1 in tumorogenesis, at least in certain tumor types. Yang et al. discovered that Stim1 and Orai1 involved in the invasiveness and metastasis of breast cancers [24]. Chen et al. obtained qualitatively similar results regarding Stim1 and Orai1 in cervical cancer growth, migration, and angiogenesis [26]. Coincidentally, Limnander et al. recently demonstrated that over-expression of SOCE sensitized DT40 B cells to both B cell antigen receptor (BCR)-induced apoptosis and thapsigargin-induced apoptosis, indicating that SOCE is a limiting factor in the induction of apoptosis during B cell development [34]. In our present study, we provided evidence for the possible contribution of SOCE to the growth of human MM cells, based on the findings that Stim1/Orai1-silencing and inhibitors of SOCE (SKF96365, 2-APB, DES) in MM cells significantly inhibited cell proliferation, and increased cell apoptosis, respectively. Our results showed that Stim1/Orai1-silencing and inhibitors of SOCE in MM cells leads to a decrease in proliferation by arresting the cell cycle at the G2/M phases. Previous research suggests that reduced SOCE has been linked to cell apoptosis due to decreased vesicle trafficking to the plasma membrane [35]. One possible explanation for these findings are the presence of enhanced $\mathrm{Ca}^{2+}$-dependent apoptotic signaling in the rescued Stim1/Orai1over expressing clone. A rise in the cytosolic $\mathrm{Ca}^{2+}$ concentration can trigger cell proliferation as well as stimulate enzymes that execute apoptotic cell death [36]. $\mathrm{Ca}^{2+}$ entry also plays a vital role in enzyme activation, cell cycle G2/M phase progression, activation of critical signal transduction pathways [37]. While, it is interesting that TP-induced SOCE is not completely abolished, neither in Orai1 nor in Stim1 deficient cells. According to previous studies, we conjecture that there may be some other subtypes of SOC channels that can affect SOCE, which deserves further study. In recent years, great progress has been made in the characterization of multiple myeloma both cytogenetic and molecular, but we are still looking for new prognostic factors, which would be a useful tool for diagnosis and prognosis of MM. Previous study found that Orai1 enhances cell proliferation and is associated with poor prognosis in some solid tumor [38]. We first analysis if Stim1/Orail expression levels can affect the outcome of MM patients. We find that the expression levels of Stim1/Orail in stage III patients were obviously higher than stage II and I patients and predicts worse prognosis, those with increased levels of Stim1/Orail expression showed shorter survival period. Therefore, the detection of Stim1/Orail may play a role in the prediction of MM patient's prognosis.

\section{Conclusion}

We demonstrated that Stim1 and Orai1 are all highly-expressed in MM tissue and cell lines. The molecular mechanism by which Stim1/Orai1 affects cancer malignant behaviors involves the regulation of $\mathrm{Ca}^{2+}$ signaling essential for cancer cell proliferation, apoptosis, and cell cycle. We also found that, in MM patients, highly-expression of Stim1/Orai1 was found in the majority of patients, and the level of Stim1/Orai1 is closely associated with the clinical outcome of MM. These results make Stim1/Orai1-mediated signaling an attractive target for therapeutic intervention. Furthermore, molecular therapy approaches targeting SOCE channels could be developed for MM patients with high malignant risk. 


\section{Cellular Physiology Cell Physiol Biochem 2018;48:2273-2285 \begin{tabular}{ll|l} 
and BiOChemistry & $\begin{array}{l}\text { DOI: 10.1159/000492645 } \\
\text { Published online: 15 August, } 2018\end{array}$ & $\begin{array}{l}\text { (c) } 2018 \text { The Author(s). Published by S. Karger AG, Basel } \\
\text { www.karger.com/cpb }\end{array}$
\end{tabular}}

Wang et al.: Orai1 and Stim1 Expression and SOCE in MM

\section{Acknowledgements}

This work was supported by the Second Affiliated Hospital of Harbin Medical University. This work was supported by grants from the Youth Science Fund of the Natural Science Foundation of China (81001051), Postdoctoral Science Foundation of China (2015M580270), Postdoctoral Science Foundation of Heilongjiang Province (LBH-Z15129), and the Young and middle-aged Science Foundation of Harbin Medical University(KYCX2018-15).

\section{Disclosure Statement}

The authors declare to have no competing interests.

\section{References}

$>1$ Lonial S, Durie B, Palumbo A, San-Miguel J: Monoclonal antibodies in the treatment of multiple myeloma: current status and future perspectives. Leukemia 2015;30:526-535.

-2 Chen R, Wang Y, Luan C, Gao C, Zhang XP, Chen BA: Effect of pomalidomide on relapsed/refractory multiple myeloma: a systematic review and meta-analysis. J Cancer 2017;8:1801-1808.

-3 Spicka I: Advances in multiple myeloma therapy during two past decades. Comput Struct Biotechnol J 2014;10:38-40.

-4 De IPP, Muz B, Azab F, Luderer M, Azab AK: Molecularly targeted therapies in multiple myeloma. Leuk Res Treat 2014;1:976567.

$>5$ Costello C: An update on the role of daratumumab in the treatment of multiple myeloma. Ther Adv Hematol 2017;8:28-37.

-6 Maiso P, Huynh D, Moschetta M, Sacco A, Al-jawai Y, Mishima Y, Asara JM, Roccaro AM, Kimmelman AC, Ghobrial IM: Metabolic signature identifies novel targets for drug resistance in Multiple Myeloma. Cancer Res 2015;75:2071-2082.

7 Berridge MJ, Bootman MD, Roderick HL: Calcium signalling: dynamics, homeostasis and remodelling. Nat Rev Mol Cell Biol 2003;4:517-529.

$>8$ Yu W, Honisch S, Schmidt S, Yan J, Schmid E, Alkahtani S, AlKahtane AA, Alarifi S, Stournaras C, Lang F: Chorein Sensitive Orai1 Expression and Store Operated $\mathrm{Ca}^{2+}$ Entry in Rhabdomyosarcoma Cells. Cell Physiol Biochem 2016;40:1141-1152.

-9 Parekh AB: Store-operated CRAC channels: function in health and disease. Nat Rev Drug Discov 2010;9:399-410.

10 Lewis RS: The molecular choreography of a store-operated calcium channel. Nature 2007;446:284-287.

11 Liou J, Kim ML, Heo WD, Jones JT, Myers JW, Ferrell JE, Meyer T: STIM is a $\mathrm{Ca}^{2+}$ sensor essential for $\mathrm{Ca}^{2+}$ store-depletion-triggered Ca ${ }^{2+}$ influx. Curr Biol 2005;15:1235-1241.

$>12$ Zhang SL, Yu Y, Roos J, Kozak JA, Deerinck TJ, Ellisman MH, Stauderman KA, Cahalan MD: STIM1 is a Ca ${ }^{2+}$ sensor that activates CRAC channels and migrates from the $\mathrm{Ca}^{2+}$ store to the plasma membrane. Nature 2005;437:902-905.

13 Potier M, Trebak M: New developments in the signaling mechanisms of the store-operated calcium entry pathway. Pflugers Arch 2008;457:405-415.

14 Feske S: ORAI1 and STIM1 deficiency in human and mice: roles of store-operated $\mathrm{Ca}^{2+}$ entry in the immune system and beyond. Immunol Rev 2009;231:189-209.

15 Johnstone LS, Graham SJ, Dziadek MA: STIM proteins: integrators of signalling pathways in development, differentiation and disease. J Cell Mol Med 2010;14:1890-1903.

16 Hogan PG, Lewis RS, Rao A: Molecular Basis of Calcium Signaling in Lymphocytes: STIM and ORAI. Annu Rev Immunol 2010;28:491-533.

17 Matsumoto M, Fujii Y, Baba A, Hikida M, Kurosaki T, Baba Y: The Calcium Sensors STIM1 and STIM2 Control B Cell Regulatory Function through Interleukin-10 Production. Immunity 2011;34:703-714. 


\section{Cellular Physiology Cell Physiol Biochem 2018;48:2273-2285 \begin{tabular}{l|l} 
and Biochemistry & DOI: 10.1159/000492645 \\
(c) 2018 The Author(s). Published by S. Karger AG, Basel \\
www.karger.com/cpb
\end{tabular}

Wang et al.: Orai1 and Stim1 Expression and SOCE in MM

18 Braun A, Vogtle T, Vargaszabo D, Nieswandt B: STIM and Orai in hemostasis and thrombosis. Front Biosci 2011;16:2144-2160.

19 Zhang W, Trebak M: STIM1 and Orai1: novel targets for vascular diseases? Sci China Life Sci 2011;54:780785.

20 Nishiyama M, Hoshino A, Tsai L, Henley JR, Goshima Y, Tessier-Lavigne M, Poo MM, Hong K: Cyclic AMP/ GMP-dependent modulation of $\mathrm{Ca}^{2+}$ channels sets the polarity of nerve growth-cone turning. Nature 2003;423:990-995.

21 Chen JP, Luan Y, You CX, Chen XH, Luo RC, Li R: TRPM7 regulates the migration of human nasopharyngeal carcinoma cell by mediating $\mathrm{Ca}^{2+}$ influx. Cell Calcium 2010;47:425-432.

$\checkmark 22$ El BC, Bidaux G, Enfissi A, Delcourt P, Prevarskaya N, Capiod T: Capacitative calcium entry and transient receptor potential canonical 6 expression control human hepatoma cell proliferation. Hepatology 2008;47:2068-2077.

-23 Holmuhamedov E, Lewis L, Bienengraeber M, Holmuhamedova M, Jahangir A, Terzic A: Suppression of human tumor cell proliferation through mitochondrial targeting. FASEB J 2002;16:1010-1016.

24 Yang S, Zhang JJ, Huang XY: Orai1 and STIM1 are critical for breast tumor cell migration and metastasis. Cancer Cell 2009;15:124-134.

25 Vanden AF, Shuba Y, Roudbaraki M, Lemonnier L,Vanoverberghe K, Mariot P, Skryma R, Prevarskaya $\mathrm{N}$ : Store-operated $\mathrm{Ca}^{2+}$ channels in prostate cancer epithelial cells: function, regulation, and role in carcinogenesis. Cell Calcium 2003;33:357-373.

26 Chen YF, Chiu WT, Chen YT, Lin PY, Huang HJ, Chou CY, Chang HC, Tang MJ, Shen MR: Calcium store sensor stromal-interaction molecule 1-dependent signaling plays an important role in cervical cancer growth, migration, and angiogenesis. Proc Natl Acad Sci USA 2011;108:15225-15230.

-27 Gregory RB, Hughes R, Riley AM, Potter BV, Wilcox RA, Barritt GJ: Inositol trisphosphate analogues selective for types I and II inositol trisphosphate receptors exert differential effects on vasopressin-stimulated $\mathrm{Ca}^{2+}$ inflow and $\mathrm{Ca}^{2+}$ release from intracellular stores in rat hepatocytes. Biochem J 2004;381:519-526.

-28 Yan J, Zhang B, Hosseinzadeh Z, Lang F: Down-Regulation of Store-Operated $\mathrm{Ca}^{2+}$ Entry and $\mathrm{Na}^{+} \mathrm{Ca}^{2+}$ Exchange in MCF-7 Breast Cancer Cells by Pharmacological JAK3 Inhibition. Cell Physiol Biochem 2016;38:1643-1651.

29 Roberts-Thomson SJ, Peters AA, Grice DM, Monteith GR: ORAI-mediated calcium entry: Mechanism and roles, diseases and pharmacology. Pharmacol Ther 2010;127:121-130.

-30 Stagno MJ, Zacharopoulou N, Bochem J, Tsapara A, Pelzl L, Al-Maghout T, Kallergi G, Alkahtani S, Alevizopoulos K, Dimas K, Calogeropoulou T, Warmann SW, Lang F, Schmid E, Stournaras C: Istaroxime Inhibits Motility and Down-Regulates Orai1 Expression, SOCE and FAK Phosphorylation in Prostate Cancer Cells. Cell Physiol Biochem 2017;42:1366-1376.

-31 Salido GM, Sage SO, Rosado JA: Biochemical and functional properties of the store-operated $\mathrm{Ca}^{2+}$ channels. Cell Signal 2009;21:457-461.

-32 Landowski TH, Megli CJ, Nullmeyer KD, Lynch RM, Dorr RT: Mitochondrial-mediated disregulation of $\mathrm{Ca}^{2+}$ is a critical determinant of Velcade (PS-341/bortezomib) cytotoxicity in myeloma cell lines. Cancer Res 2005;65:3828-3836.

-33 Motiani RK, Hyzinskigarcía MC, Zhang X, Henkel MM, Abdullaev IF, Kuo YH, Matrougui K, Mongin AA, Trebak M: STIM1 and Orai1 Mediate CRAC Channel Activity and are Essential for Human Glioblastoma Invasion. Pflugers Arch 2013;465:1249-1260.

-34 Limnander A, Depeille P, Freedman TS, Liou J, Leitges M, Kurosaki T, Roose JP, Weiss A: STIM1, PKC- $\delta$ and Ras GRP set a threshold for proapoptotic Erk signaling during B cell development. Nat Immunol 2011;12:425-433.

35 Vacher P, Vacher AM, Pineau R, Latour S, Soubeyran I, Pangault C, Tarte K, Soubeyran P, Ducret T, BressonBepoldin L: Localized Store-Operated Calcium Influx Represses CD95-Dependent Apoptotic Effects of Rituximab in Non-Hodgkin B Lymphomas. J Immunol 2015;195:2207-2215.

-36 Roderick HL, Cook SJ: $\mathrm{Ca}^{2+}$ signalling checkpoints in cancer: remodelling $\mathrm{Ca}^{2+}$ for cancer cell proliferation and survival. Nat Rev Cancer 2008;8:361-375.

37 Aggarwal BB, Sethi G, Nair A, Ichikawa H: Nuclear factor-kB: Aholygrailin cancer prevention and therapy. Curr Signal Transduct Ther 2006;1:25-52.

-38 Gui L, Wang Z, Han J, Ma H. Li Z: High Expression of Orai1 Enhances Cell Proliferation and is Associated with Poor Prognosis in Human Colorectal Cancer. Clin Lab 2016;62:1689-1698. 